\section{Handgelenk und Handwurzel \\ Anteil von Radiokarpal- und Mediokarpalgelenk an der Dart Throwing Motion}

Kane PM et al. Relative Contributions of the Midcarpal and Radiocarpal Joints to Dart-Thrower's Motion at the Wrist. J Hand Surg Am 2018; 43: $234-240$

Die normale Beweglichkeit des Handgelenks ist für eine Vielzahl von Alltagsaktivitäten notwendig. Umgekehrt können Schmerzen und Bewegungseinschränkungen des Handgelenks den Alltag erheblich beeinträchtigen. Zur ausgeprägten physiologischen Beweglichkeit der Hand tragen 4 Teilgelenke bei: distales Radioulnargelenk, Radiokarpalgelenk, Mediokarpalgelenk und zum Teil die Karpometakarpalgelenke.

Die schräge Handgelenkbewegung, die sog. Dart Throwing Motion (DTM), verläuft von der Radialextension (als Kombination von Radialabduktion und Dorsalextension) zur Ulnarflexion (aus Ulnarabduktion und Palmarflexion) und ist die häufigste im Alltag ausgeführte Aktivität des Handgelenks. Sie ist bspw. erforderlich beim Öffnen von Schraubverschlüssen, Einhämmern von Nägeln und beim Kämmen. Bisher ist aber nicht endgültig geklärt, welche Knochen- und Gelenkabschnitte v.a. zur DTM beitragen. Diese Kenntnis wäre etwa für die Physiotherapie zur Wiederherstellung der Handgelenkfunktion, die Planung von operativen Eingriffen oder die Entwicklung von Handgelenkprothesen bedeutsam. Forscher aus den USA haben nun eine weitere Untersuchung dazu vorgenommen.

Patrick Kane und seine Kollegen habe dafür an 6 Leichenarmen eine biomechanische Studie durchgeführt. Nach der Freipräparation analysierten sie in Neutralstellung des Unterarms die Beweglichkeit des Handgelenks in insgesamt 24 Freiheitsgraden unter 3 verschiedenen Bedingungen:
- am intakten Gelenk,

- bei Simulation einer radiokarpalen Fusion (entsprechend einer Teilarthrodese) und

- bei Simulation einer pankarpalen Fusion (entsprechend einer vollständigen Arthrodese aller Handwurzelknochen, nicht aber des Radiokarpalgelenks).

Für jede Bedingung und jeden Freiheitsgrad applizierten die Wissenschaftler Drehmomente von $\pm 1,5 \mathrm{Nm}$ und dokumentierten die jeweils resultierende Rotation des Handgelenks. Daraus berechneten sie anschließend das Bewegungsmuster des Gelenks (envelope of motion). Die Auswertung ergab

- in reiner Extension des Handgelenks einen

- signifikant größeren Bewegungsumfang des intakten Gelenks, mit $61,4^{\circ}\left( \pm 9,8^{\circ}\right) \mathrm{im}$ Vergleich zu $23,9^{\circ}$ $\left( \pm 17,8^{\circ}\right)$ bei pankarpaler Fusion und $24,3^{\circ}\left( \pm 12,4^{\circ}\right)$ bei radiokarpaler Fusion, jedoch

- in reiner Flexion

- keine signifikanten Unterschiede des Bewegungsumfangs zwischen den 3 Bedingungen, mit $62,1^{\circ}$ $\left( \pm 22,4^{\circ}\right)$ am intakten Gelenk, $48,4^{\circ}$ $\left( \pm 16,3^{\circ}\right)$ bei pankarpaler Fusion und $37,9^{\circ}\left( \pm 6,2^{\circ}\right)$ bei radiokarpaler Fusion.

Bei Ulnarabduktion, $15^{\circ}$ Radialextension und $15^{\circ}$ Ulnarflexion war darüber hinaus die Beweglichkeit in beiden Fusionsgruppen signifikant gegenüber dem unbehandelten Gelenk eingeschränkt. Zwischen den beiden Fusionsgruppen dagegen fanden sich für keine der 24 Bewegungsrichtungen signifikante Unterschiede im Bewegungsumfang.

Das Bewegungsmuster lag unter allen 3 Bedingungen schräg zur Achse der reinen Flexion/Extension und entsprach eher der Richtung Ulnarflexion/Radialextension.
FAZIT

Im Vergleich zum gesunden Handgelenk vermindern zwar sowohl die pankarpale Fusion als auch die radiokarpale Teilfusion den Bewegungsumfang im Gelenk, fassen die Autoren zusammen. Die beiden Fusionsgruppen unterscheiden sich untereinander dagegen nicht wesentlich. Die Hauptbewegungsrichtung des Gelenks bei der Dart Throwing Motion wurde außerdem durch die Fusionen zumindest im Ex-vivo-Modell - trotz des verminderten Gesamtbewegungsumfangs - nicht wesentlich beeinträchtigt.

Dr. Elke Ruchalla, Bad Dürrheim 